\title{
Maxillary Osteomyelitis with Candidiasis due to Extraction in Uncontrolled Diabetes State-A Case Report
}

\author{
Laxmi Shetty, Deepak Kulkarni, Archana Anshuman Gupta* and Bhushan Gawande \\ Department of Oral Pathology and Microbiology, Dr. D.Y. Patil Dental College and Hospital, Pune, Maharashtra, India
}

\begin{abstract}
Background: The uncontrolled diabetes is fast growing epidemic in India. This disease if missed before extraction will cause the patient to lose their maxillary bone as reported in this case report. The opportunistic candida infection was giving the diagnostic dilemma. The radiographs revealed osteolytic changes with destruction of the left maxilla. Sequestrum due to osteomyelitis was diagnosed and sequestrectomy was performed. The uncontrolled diabetes status improved post operatively only after sequestrum was removed. The patient was rehabilated with an obturator for the left maxillary region.
\end{abstract}

Case description: A 56 year female patient had undergone extraction in uncontrolled diabetes state at a local dentist who reported at our department with osteomyelitis and candidiasis of left maxilla. There was a situation of diagnostic dilemma for treating the uncontrolled diabetes and surgical management. The computed tomography revealed lytic destruction of the left maxilla. Sequestrectomy was the solution which reduced her blood sugar level and healing occured uneventfully.

Conclusion: An uncontrolled diabetic state is always a relative contraindication forextraction. The patient's blood sugar level should be in controlled state before extraction. The osteomyelitis with candidiasis was induced due to extraction causing the destruction of left maxilla. Sequestrectomy cured the patient of the progressive osteomyelitis. It is of paramount significance that the negligence by the local dentist who performed the extraction in uncontrolled diabetic condition caused the loss of the left maxilla. The effective case history taken prior to extraction would have prevented the patient from all complications.

Keywords: Maxillary osteomyelitis; Diabetes; Candidiasis; Sequestrectomy

\section{Introduction}

The World Health Organization (WHO) estimates that nearly 200 million people all over the world suffer from diabetes and this number is likely to be doubled by 2030. In 2011 there were 366 million people with diabetes globally, and this is expected to rise to 552 million by 2030 [1]. Diabetes is a condition primarily defined by the level of hyperglycemia giving rise to risk of microvascular damage (retinopathy, nephropathy and neuropathy) [2]. A dental practitioner in Indian scenario should not neglect the case history especially of diabetes before performing an extraction in a rural center since it may jeopardize the bone causing complications. Type 2 diabetes mellitus (T2DM), by definition is a heterogeneous, multifactorial, polygenic syndrome which results from insulin receptor dysfunction [3]. The studies show that diabetes mellitus patients have a higher risk of death than people without diabetes mellitus [4-6]. This is a case report of a female patient who had to undergo sequestrectomy (SE) due to maxillary osteomyelitis (MO) of left upper alveolus and maxilla since her dentist had performed extraction in uncontrolled diabetic state. Osteomyelitis is considered as "an inflammatory condition of the bone that usually begins as an infection of the medullary cavity, rapidly involves the harversian systems and quickly extends to the periosteum of the area" [7]. This is a paper trying to reach out to the dental practitioners highlighting the importance of uncontrolled diabetes mellitus (UDM) causing a challenge to the oral and maxillofacial surgeon and anesthesiologists in a case of misdiagnosis. The extraction of the teeth in T2DM state presented with opportunistic candida infection and osteomyelitis [8] of the left alveolar and maxillary region with non-healing sockets.

\section{Case History}

A 56 year old female patient reported to department of Oral and Maxillofacial surgery, Dr.D.Y.Patil Dental College and Hospital, Pune with pain and swelling in the left side of the upper jaw (Figure 1). The case history revealed that patient had a history of extraction 6 months back. This particular extraction of left posterior teeth 26,27 was done by a local dentist at her home town. The dentist had not taken her history of UDM and performed the extraction. The patient after reporting to our department underwent extensive investigations to assess the blood sugar level. She was not on any antihypoglycemics and recorded a fasting blood sugar level of $380 \mathrm{mg} / \mathrm{dl}$.

On local examination the non-healing socket of 26, 27 was evident with fever, severe pain ,tenderness and inflammation in the area (Figure 2). The buccal and palatal gingival showed signs of non-healing with necrotic bone exposed. The superficial area had white crusts on the area of the attached gingival region of extracted area of 26, 27. The patient was administered intravenous antibiotics.

The computed tomography (Figure 3 ) of the area revealed extensive irregular lytic destruction of left alveolar process of left maxilla, anterior floor of left maxillary sinus with minimal soft tissue, dense sclerosis of zygomatic process of left maxilla. Bony discontinuity was noted in posterolateral wall of left maxillary sinus with an impression of sequestrum formation.

*Corresponding author: Archana Anshuman Gupta, MDS, Department of Oral Pathology and Microbiology, Dr. D.Y. Patil Dental College and Hospital, C - 1204, MSR Queenstown, Behind Lokmanya Hospital, Chinchwad, Pune411033, Maharashtra, India, Tel: +917276072737, +917276072735; E-mail: archana.gupta@dpu.edu.in

Received December 20, 2014; Accepted January 08, 2015; Published January 14,2015

Citation: Shetty L, Kulkarni D, Gupta AA, Gawande B (2015) Maxillary Osteomyelitis with Candidiasis due to Extraction in Uncontrolled Diabetes State-A Case Report. Dentistry 5: 279. doi:10.4172/2161-1122.1000279

Copyright: $\odot 2015$ Shetty L, et al. This is an open-access article distributed unde the terms of the Creative Commons Attribution License, which permits unrestricted use, distribution, and reproduction in any medium, provided the original author and source are credited. 


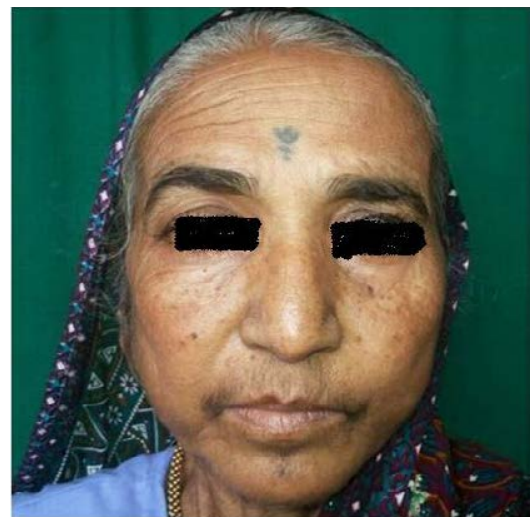

Figure 1: Preoperative view.

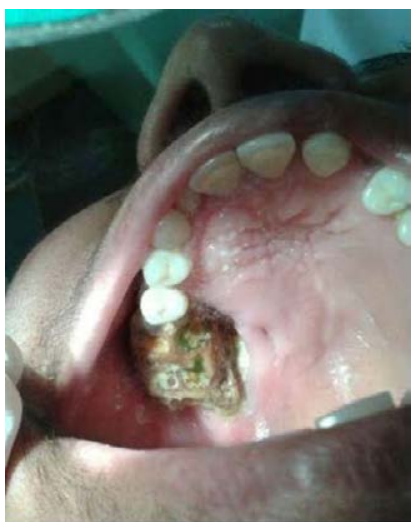

Figure 2: Intraoral view with exposed necrotic bone.

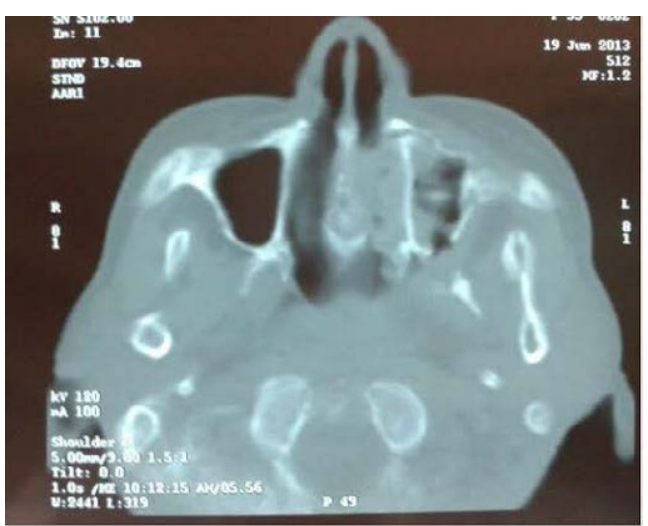

Figure 3: Computed Tomography showing lytic destruction of bone in left maxillary region.

The cytological smear of the white deposits on the alveolar process revealed candidiasis. The blood sugar level remained uncontrolled and injectable insulin was started. There was erratic variation in fasting and random blood sugar levels. The intravenous antifungal therapy was started and the candida growth was controlled. The necrosis of the bone increased and hence it was decided for sequestrectomy of left maxilla under general anesthesia with high risk consent.

The sequestrum of the left maxilla was removed with the extraction of the 25 (Figures 4 and 5). The area was packed with betadine gauze and acrylic plate was given to aid healing. The healing was uneventful within 15 days after the surgery. The patient was rehabilated with a left maxillary obturator (Figure 6).

\section{Discussion}

India leads the world with largest number of diabetic subjects earning the dubious distinction of being termed the "diabetes capital of the world" [9]. Unfortunately the poor diabetic subjects delay taking treatment leading to increased risk of complications [10]. The preoperative assessment is the primary responsibility of the dental practitioner and many of the complications can be prevented by a thorough preoperative evaluation and strict control of blood glucose levels during the preoperative period. Our case presents here as a state of exposed, necrotic bone in the region of the maxillary alveolar region because of extraction done in the UDM state by her local dentist. The patient presented with T2DM under no medication.

Diabetes is a chronic disease characterized by the body's inability to

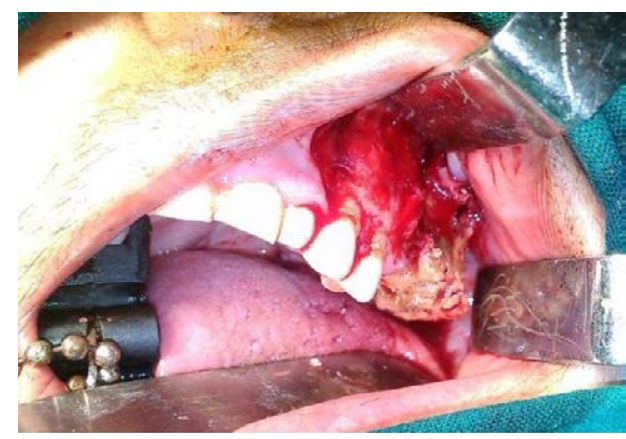

Figure 4: Intraoperative view.

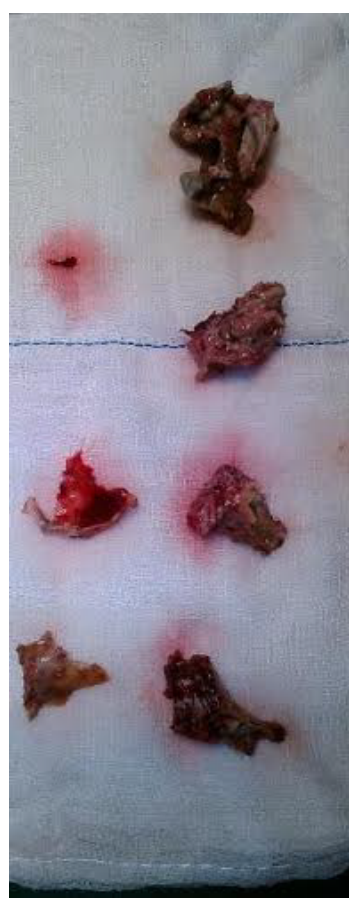

Figure 5: Sequestrum. 


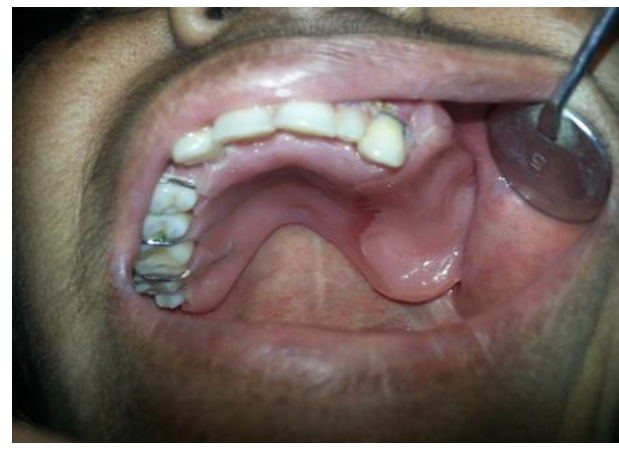

Figure 6: Postoperative view with obturator.

process blood glucose properly. Type 2 diabetes is a complex metabolic disorder resulting from decreased pancreatic insulin secretion and variable contributions of decreased insulin action or insulin resistance in target tissues, mainly muscle and the liver [11].

The patients who have poorly controlled diabetes are also predisposed to impair wound healing. Operative injury or direct infection from the teeth or the antrum is the usual etiological factor for the maxillary osteomyelitis [12]. In this case the UDM was the cause of the impaired wound healing after extraction. Diabetes induced impaired wound healing is characterized by inhibition of the inflammatory response to wounding, macrophage infiltration, angiogenesis, fibroplasias, reparative collagen accumulation and wound breaking strength [13]. Macro vascular disease and microvascular dysfunction may result in compromised local circulation, leading to delayed response to infection [14].

$\mathrm{MO}$ is relatively uncommon in comparison to mandible due to the porous nature of the bone, significant collateral blood flow and thin cortices [15].

This case reported with fever, malaise, pain in the area with a non-healing socket and exposed bone. These characterize chronic osteomyelitis (CO). The challenging part was the cytological smear of the area which showed hyphae suggestive of candidiasis. She underwent intravenous antifungal therapy. The maxilla rarely undergoes necrosis, due to its rich vascularity. Maxillary necrosis can occur due to bacterial infections such as osteomyelitis, viral infections such as herpes zoster or fungal infections such as mucormycosis, aspergillosis, etc. [16]. Kumar et al. have reported colonization and carriage of candida in the oral cavity was found to be higher in diabetic subjects than in nondiabetic subjects [17].

The patient presented with no diet control or antihypoglycemic agents when she reported to our department. For blood glucose levels of 301 to $350 \mathrm{mg} / \mathrm{dl}, 6$ to $8 \mathrm{U}$ of regular human insulin may be given subcutaneously. For a level more than $350 \mathrm{mg} / \mathrm{dl}$, the surgeon may use an insulin infusion to correct the hyperglycemia, or should consider canceling the surgery to stabilize the blood glucose levels and complete a more extensive workup focusing on the cause of the poor control [18]. The patient was treated for UDM state with human insulin. The diet counseling was done. The computed tomography reported lytic areas of destruction in the left maxillary region which have also reported by Alberto [19]. Among the skull bones, the CO is more frequently observed in the mandible and, in fewer cases, in the maxilla, but generally limited to one anatomic site as mentioned by Lew [20], although it may spread out to other areas, especially in diabetic, immunosuppressed and hospitalized patients as recorded by
Brady [21]. The radiographic characteristics of the osteolysis observed in the chronic osteomyelitis of the maxilla and mandible are more evident in younger patients; meanwhile the bone condensation is more frequent in adults, particularly in lesions of the mandible [22]. The treatment of these infections depends on the SE, surgical debridement of the wound, and removal of the bone cortex, associated to the systemic use of antimicrobials has been evaluated by Lew [20]. In our case we have followed the same line of treatment with planned SE of the left maxillary region with extraction of upper left second premolar. The histopathological examination confirmed sequestrum as seen in all CO. The insulin infusion was given intraoperatively and changed to oral antihypoglycemic agents after healing occurred without any complications. In cases of maxillary osteomyelitis which resulted in a maxillary defect following surgical treatment leading to oroantral fistula, we found that the obturators were the favored method of filling the defect as mentioned by Barry. The entire maxilla of the left region was removed and replaced with obturator after which healing occurred uneventfully.

\section{Conclusion}

The meticulous perioperative management of diabetes has been responsible for the healing in the operated site and resolution of CO. In India, there are nearly 50 million diabetics, according to the statistics of the International Diabetes Federation. As the incidence of diabetes is on the rise, doctors say, there is a proportionate rise in the complications that are associated with diabetes. They point out that it is a very crucial stage and awareness on the part of people and administration about diabetes is very essential, adding that people should be made aware and educated about their health and fitness level to reduce the number of patients in India. This case will set awareness since the need for appropriate perioperative management of diabetes will certainly prevent CO and loss of the maxilla. DM affects many patients who seek oral and maxillofacial surgery hence meticulous perioperative management are essential in patients undergoing surgical procedures.

\section{References}

1. Whiting DR, Guariguata L, Weil C, Shaw J (2011) IDF diabetes atlas: global estimates of the prevalence of diabetes for 2011 and 2030. Diabetes Res Clin Pract 94: 311-321.

2. World Health Organization (1999) Definition, Diagnosis and Classification of Diabetes Mellitus and its Complications. Report of a WHO Consultation, Geneva.

3. Banerjee M, Vats P (2014) Reactive metabolites and antioxidant gene polymorphisms in Type2 diabetes mellitus. Redox Biology 2:170-177.

4. Roper NA, Bilous RW, Kelly WF, Unwin NC, Connolly VM (2002) South Tees Diabetes Mortality Study (2002) Cause-specific mortality in a population with diabetes: South Tees Diabetes Mortality Study. Diabetes Care 25: 43-48.

5. Gregg EW, Gu Q, Cheng YJ, Narayan KM, Cowie CC (2007) Mortality trends in men and women with diabetes, 1971 to 2000.Ann Intern Med 147: 149-155.

6. Morgan CL, Currie CJ, Peters JR (2000) Relationship between diabetes and mortality: a population study using record linkage.Diabetes Care 23: 11031107.

7. Prasad KC, Prasad SC, Mouli N, Agarwal S (2007) Osteomyelitis in the head and neck.Acta Otolaryngol 127: 194-205.

8. Hudson JW (1993) Osteomyelitis of the jaws: a 50-year perspective.J Oral Maxillofac Surg 51: 1294-1301.

9. Mohan V, Sandeep S, Deepa R, Shah B, Varghese C (2007) Epidemiology of type 2 diabetes: Indian scenario. Indian J Med Res 125: 217-230.

10. Ramachandran A, Snehalatha C, Vijay V, King H (2002) Impact of poverty on the prevalence of diabetes and its complications in urban southern India Diabet Med 19: 130-135. 
Citation: Shetty L, Kulkarni D, Gupta AA, Gawande B (2015) Maxillary Osteomyelitis with Candidiasis due to Extraction in Uncontrolled Diabetes State-A Case Report. Dentistry 5: 279. doi:10.4172/2161-1122.1000279

11. Inzucchi SE (2002) Oral antihyperglycemic therapy for type 2 diabetes: scientific review. JAMA 287: 360-372.

12. Macbeth $\mathrm{R}$ (1952) Osteomyelitis of the maxilla. J Laryng OtolM 66: 1369-1370.

13. Qiu JG, Chang TH, Steinberg JJ (1998) Single local instillation of Staphylococcus aureus peptidoglycan prevents diabetes-induced impaired wound healing. Wound Repair Regen 6: 449-456.

14. Goodson WH 3rd, Hunt TK (1979) Wound healing and the diabetic patient Surg Gynecol Obstet 149: 600-608.

15. Wannfors K, Gazelius B (1991) Blood flow in jaw bones affected by chronic osteomyelitis. Br J Oral Maxillofac Surg 29: 147-153.

16. Auluck A (2007) Maxillary necrosis by mucormycosis. a case report and literature review. Med Oral Patol Oral Cir Bucal 12: E360-364.

17. Kumar BV, Padshetty NS, Bai KY, Rao MS (2005) Prevalence of Candida in the oral cavity of diabetic subjects. J Assoc Physicians India 53: 599-602.
18. Plodkowski RA, Edelman SV (2001) Pre-surgical evaluation of diabetic patients. Clin Diabetes 19: 92-95.

19. Fish AW, Espinosa C, Riva Parra V,Quezadall SR (2012) Upper jaw chronic osteomyelitis.Report of four clinical cases. Revista Odontológica Mexicana 16: 105-111.

20. Lew DP, Waldvogel FA (2004) Osteomyelitis. Lancet 364: 369-379.

21. Brady BA, LeiD JG, Costerton JW, Shirtliff ME (2006) Osteomyelitis: clinical overview and mechanisms of infection persistence. Clin Microbiol Newsletter 28: 65-72.

22. Gaetti-jardim EJ, Ciesielski FIN, Possagno R, Castro AL, Marqueti AC, et al (2010) Chronic osteomyelitis of the maxilla and mandible:microbiological and clinical aspects. Int J Odontostomat 4: 197-202. 